\title{
Dynamic dielectric properties and the $\gamma$ transition of bromine doped polyacrylonitrile
}

\author{
N. Cohen ${ }^{1}$, A. Greenbaum ${ }^{2}$, Y. Feldman'2, G. Marom ${ }^{*}$ \\ ${ }^{1}$ Casali Institute of Applied Chemistry, The Institute of Chemistry, The Hebrew University of Jerusalem, \\ 91904 Jerusalem, Israel \\ 2Department of Applied Physics, School of Computer Science and Engineering, The Hebrew University of Jerusalem, \\ 91904 Jerusalem, Israel
}

Received 16 July 2007; accepted in revised form 29 August 2007

\begin{abstract}
Based on monitoring the $\gamma$ process (the lowest temperature-relaxation) in polyacrylonitrile (PAN) by dynamic dielectric spectroscopy, new evidence for the formation of a charge transfer complex between bromine dopants and nitrile groups is presented. The experimental work is carried out on PAN and nitrile polymerized PAN with and without bromine doping and the effects of these factors on the $\gamma$ process are measured. Nitrile polymerization results in diminishing of the $\gamma$ process and in a $15 \%$ increase in its activation energy, whereas bromine doping produces splitting of the original $\gamma$ process in PAN - coupled with a significant activation energy increase - and its complete disappearance in nitrile polymerized PAN. Both the splitting of the $\gamma$ process and the higher activation energy reflect bromine-nitrile adduct formation.
\end{abstract}

Keywords: smart polymers, polyacrylonitrile (PAN), charge transfer bromine complexes, dynamic dielectric behavior, $\gamma$ transition

\section{Introduction}

Polyacrylonitrile is an attractive polymer because of its ability to form long conjugated imine chains (conjugated PAN) via polymerization of its pending nitrile groups, by heating above $180^{\circ} \mathrm{C}$. Also formed by the nitrile polymerization is a condensed cyclic structure, referred to by the term 'ladder polymer', which increases the thermal stability of the system [1]. The conjugated $\pi$ system renders PAN an outstanding candidate for various applications that involve semi-conductive properties. The ability of PAN to form a conjugated imine system of potential electrical conductivity had intrigued our research for quite some time prior to the present study. We demonstrated earlier that, considering the analogy to polyacetylene and other conductive polymers, it was reasonable to expect that doping

*Corresponding author, e-mail: gadm@vms.huji.ac.il

(c) BME-PT and GTE of PAN with acceptor-type compounds would be significantly beneficial for its electrical properties [2]. Conceivably, as in polyacetylene, doping (oxidation) with iodine or bromine causes electrons of the conjugated systems to be ejected from the polymer, leaving positive charge 'holes' that can move along the chain.

Unlike polyacetylene, however, both PAN and conjugated imine systems exhibit a more complex state due to the presence of nitrile/imine nitrogen that can donate its lone electron pair to the acceptor. Thus, acceptor type compounds and ions such as halogens, $\mathrm{Ag}^{+}, \mathrm{FeCl}_{3}$, and $\mathrm{CuCl}_{2}$ can form coordination bonds with the cyano/imine nitrogen of PAN, wherein a lone electron pair from occupied $2 p$ orbitals is donated to the empty $s$ orbitals of the acceptor to form a $\delta$-bond. The coordination of cyano nitrogen and $\mathrm{Ag}^{+}$was shown to be highly 
stable and to endure the reduction of silver ions in a process to form metallic silver nanoparticles free of aggregation [3]. The participation of nitrile/imine groups in coordination bonds with carbon nanotubes was also studied recently by our group, showing that the $\pi$ interactions were stronger than the original dipole-dipole interactions of the nitriles [4]. It was claimed that in view of the strong metallic character of carbon nanotubes, the role of the nitrile groups is reversed and they act as an electron acceptor, unlike their role as electron donor in the formation of stable electron donoracceptor (EDA) complexes with Lewis acids [5]. This present work studies the behavior of doped PAN and the ability of halogens, such as $\mathrm{Br}_{2}$, to create charge transfer complexes/adducts with the conjugated system and the nitrile groups. In this communication we present corroborating evidence for a coordination bond that forms between the nitrile/imine nitrogen of $\mathrm{PAN}$ and $\mathrm{Br}_{2}$. This evidence resulted from a study of molecular processes and electronic properties of PAN doped with bromine, which was based on frequency and temperature-dependent complex dielectric permitivity [6]. Accordingly, the new evidence is based on mobility changes due to doping by bromine reflected in a second order phase transition (the $\gamma$ transition) of both PAN and conjugated PAN.

Generally, amorphous or semicrystalline pending side-group polymers exhibit two or three second order phase transitions, respectively. In the semicrystalline polymers, such as polypropylene and PAN, the three transitions are designated in descending order from the melting temperature as $\alpha, \beta$, and $\gamma$ [7]. A recent paper on dynamic mechanical relaxation in PAN with different stereoregularities presents a summary of the relaxation temperatures for PAN, as reported previously in several references, and concludes by pointing out the characteristics of each transition and assigning it to corresponding molecular processes [8]. In agreement with the conventional claim that the lowest temperature transition (e.g., the $\gamma$ transition) in side-group polymers (PP, PVC, PS, etc.) is associated with local motion of groups such as $-\mathrm{CH}_{3}$ and $-\mathrm{Cl}$, the paper assigns the $\gamma$ transition in PAN to local mode motions of syndiotactic and short isotactic sequences. It is also pointed out that the lower tail of this relaxation extends down to $-150^{\circ} \mathrm{C}$.
Accordingly, the molecular processes in bromine doped PAN were characterized by dielectric measurements to test the hypothesis that if a coordination bond should form between the nitrile/imine nitrogen of $\mathrm{PAN}$ and $\mathrm{Br}_{2}$, the $\gamma$ relaxation process would be affected, perhaps by shifting to a higher temperature and exhibiting a higher activation energy.

\section{Experimental procedure}

\subsection{Materials and sample preparation}

Polyacrylonitrile powder was obtained from Scientific Polymer Products, Inc.; the polymer was characterized by a melting point of $317^{\circ} \mathrm{C}$, a glass transition temperature of $125^{\circ} \mathrm{C}$, and $M_{w} \sim$ $150000 \mathrm{~g} / \mathrm{mol}$.

Films were prepared in a solution casting method, by mixing $2 \mathrm{~g}$ of PAN powder and $25 \mathrm{ml}$ of dimethyl formamide solvent at $60^{\circ} \mathrm{C}$ for a few minutes, producing a transparent mixture. The mixture was dried in a hood for a few days and then compressed (Carver 2518 press) at $100^{\circ} \mathrm{C}$ (pressure $2 \mathrm{MPa}$ ) and then returned to the hood for final drying.

\subsection{Preparation of doped PAN}

The samples were doped with bromine, which was placed in a desiccator with the samples for various durations up to $10 \mathrm{~h}$. The samples were weighed before and after doping until the weight was stabilized and the weight change was calculated.

\subsection{Preparation of heat-treated PAN}

The samples were heat-treated in air either at $220^{\circ} \mathrm{C}$ for $30 \mathrm{~min}$ or at $250^{\circ} \mathrm{C}$ for $1.5 \mathrm{~h}$.

\subsection{Dielectric spectroscopy measurements}

Dielectric measurements were performed by using a broad band Alpha high resolution dielectric analyzer (Novocontrol) with automatic temperature control in a range of -100 to $160^{\circ} \mathrm{C}$ and at a frequency range of $0.1-10^{6} \mathrm{~Hz}$. A $3^{\circ} \mathrm{C}$ step was applied throughout the measurement routine. Each sample, with a thickness in the range of $0.01-$ $0.3 \mathrm{~mm}$, was inserted between parallel plate electrodes of the measurement cell at room temperature, and measurement was preceded by cooling 
down to $-100^{\circ} \mathrm{C}$. The measurement produced the complex dielectric permittivity $\varepsilon^{*}$ from which the real and imaginary components, namely the permittivity $\varepsilon^{\prime}$, and the dielectric loss $\varepsilon$ " were resolved.

\section{Results and discussion}

To validate the research hypothesis, four systems were tested, namely, pristine PAN, heat-treated PAN (at $250^{\circ} \mathrm{C}$ ), $\mathrm{Br}_{2}$ doped $\mathrm{PAN}$, and $\mathrm{Br}_{2}$ doped heat-treated PAN. All four systems were examined by dielectric spectroscopy and the 3-D plots of the dielectric loss $\varepsilon$ " as a function of frequency and temperature for qualitative impression are presented in Figure 1. As an example, the dielectric spectroscopy response of the pristine PAN sample is presented over the whole tested temperature range in Figure 1a. A total of three relaxation processes can be observed, designated in descend-
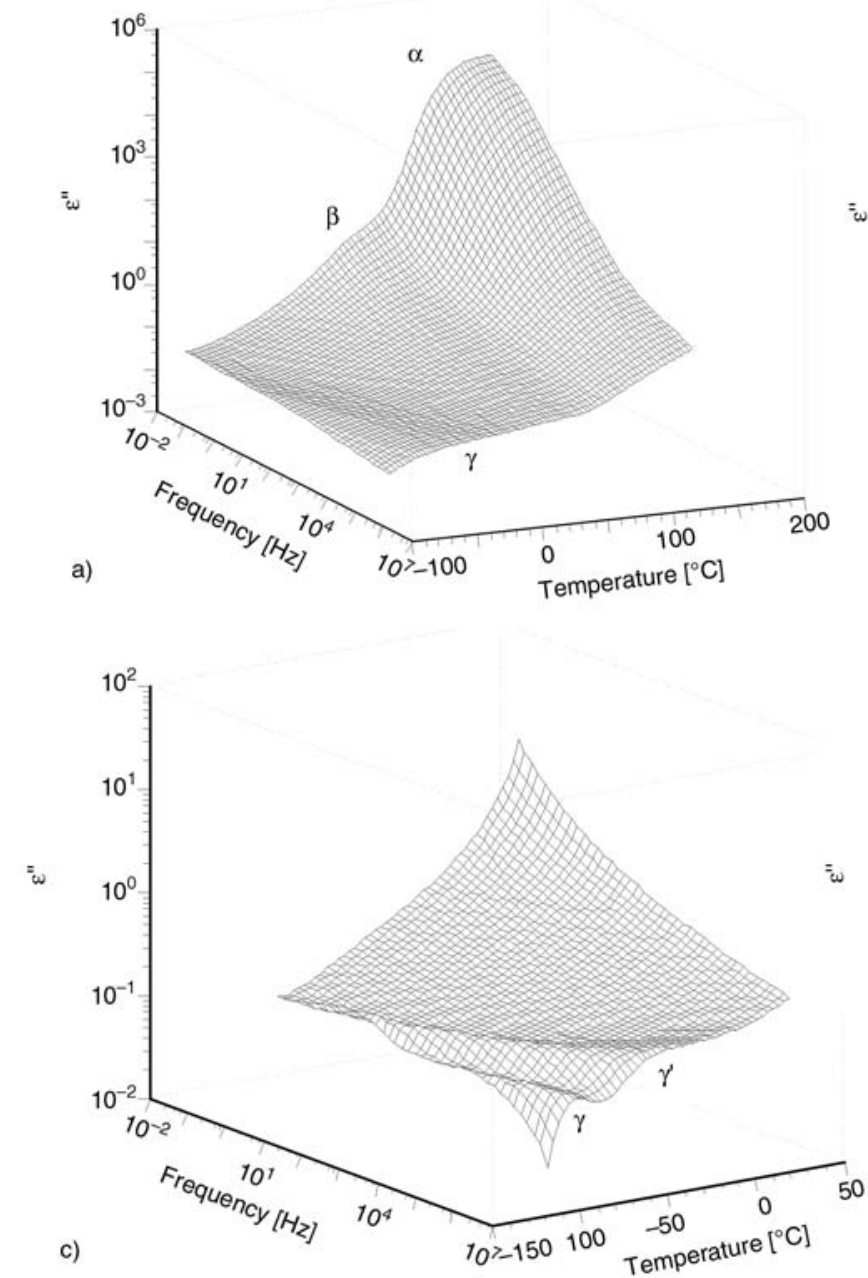

ing order (from high to low temperature) as $\alpha, \beta$, and $\gamma$ according to the common convention [7]. The $\alpha$ relaxation is assigned to the interaction between the amorphous and crystalline phases (but in fact, might be masked by the dynamic percolation process [9] - not discussed here). The $\beta$ process is situated in the temperature range $50-100^{\circ} \mathrm{C}$ and in almost the whole frequency range; it is assigned to the glass-rubber transition of the amorphous phase. The $\gamma$ process is situated in the high frequency range and in the temperature range -100 to $-30^{\circ} \mathrm{C}$ and is assigned to the motion of nitrile-side-groups. Obviously, in this study we are interested in the $\gamma$ process and how it reflects formation of a nitrile$\mathrm{Br}_{2}$ complex. Accordingly, Figures 1b-1d present the dielectric spectroscopy results of the other three samples over a lower temperature range that is limited to the $\gamma$ process regime. It is seen (in Figure 1b) that the thermal treatment that produces the conju-
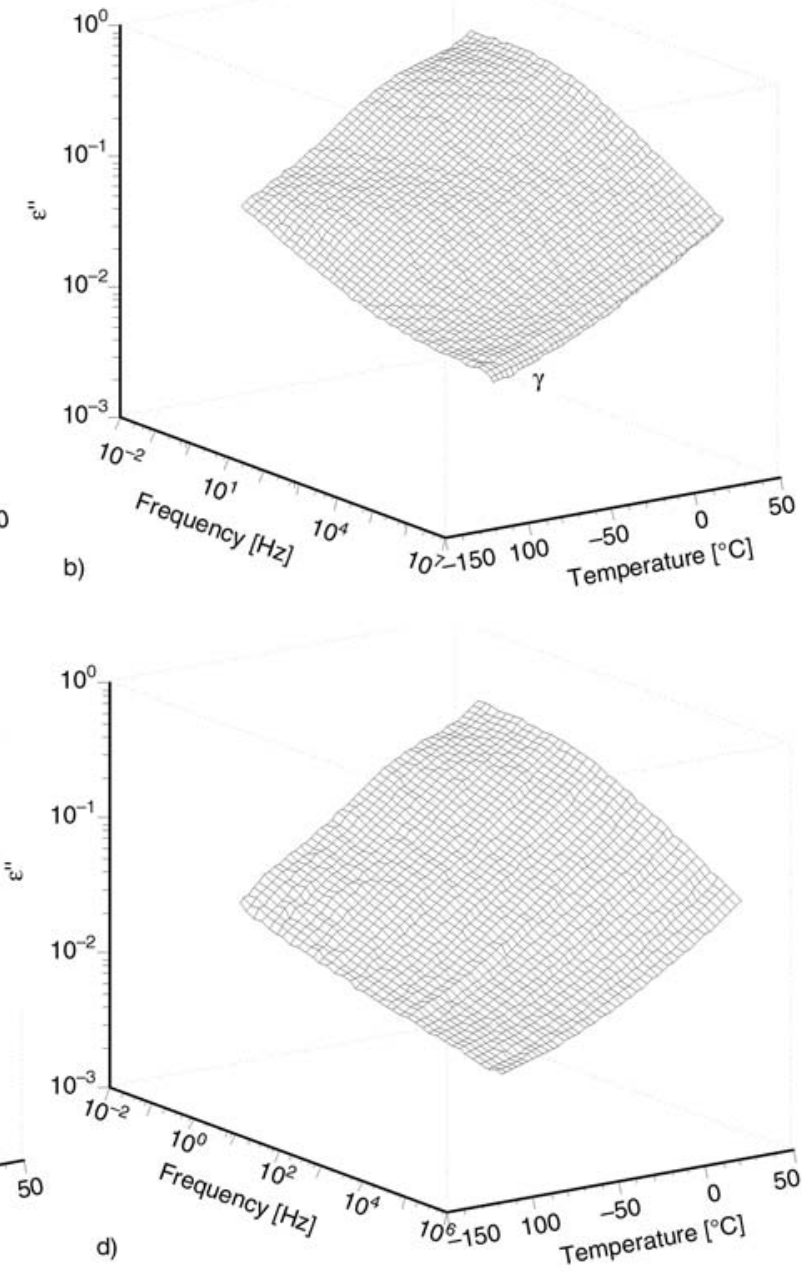

Figure 1. 3-D plots of the dielectric loss $\varepsilon$ " as a function of frequency and temperature of: (a) the pristine PAN (over the full experimental temperature range), showing the three typical relaxation processes; (b) heat-treated (at $250^{\circ} \mathrm{C}$ ) PAN; (c) bromine doped PAN; (d) bromine doped heat-treated PAN 
gated imine system as part of a ladder structure results in restriction of movement of the remaining nitrile groups and consequently in diminishing of the $\gamma$ process. (Additional evidence for the decrease in nitrile group concentration and their partial polymerization to form a conjugated imine system was received by FTIR analysis [6].)

Doping of PAN with Br was monitored by recording the weight increase and by $\mathrm{X}$-ray photoelectron spectroscopy (XPS) analysis of the proportion of ionic/covalent $\mathrm{Br}$ as a function of exposure time, as done in our previous study of $\mathrm{Br}$ treated graphite nanoplatelets [10]. However, because at this stage we were unable to identify consistent trends, only the results of 8 and $10 \% \mathrm{Br}$ weight increase, respectively, are reported. It is seen that doping of PAN with $\mathrm{Br}$ results in splitting of the $\gamma$ process peak into two peaks denoted by $\gamma$ and $\gamma^{\prime}$ (Figure 1c). As an example, the split $\gamma$ peak is shown again in Figure 2, where 2-D plots of the dielectric loss as a function of temperature are shown for the $10^{4} \mathrm{~Hz}$ frequency. Apparently, a certain proportion of the nitrile groups have formed complexes with the bromine, producing bulkier and more hindered side group adducts. Their movement requires higher free volume, thus the $\gamma$ ' process, thought to substitute the original $\gamma$ process, is shifted to a higher temperature. The new $\gamma$ process in the bromine doped PAN is situated at a lower temperature relative to the original one. This new $\gamma$ process is assigned to $-\mathrm{Br}$ side groups formed by bromination of the polymer backbone (see Figure 3 - the proportion of $\mathrm{C}-\mathrm{Br}$ covalent bonds as a function of

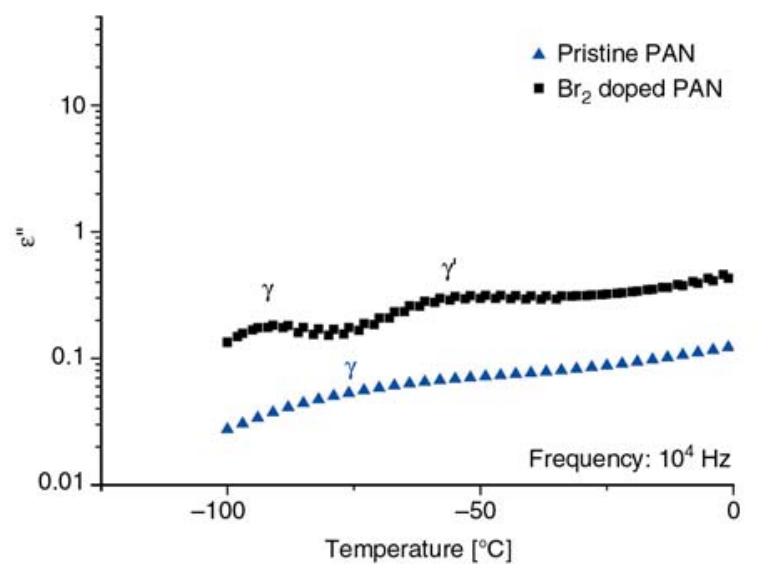

Figure 2. 2-D plots of the dielectric loss $\varepsilon$ " as a function of temperature for a constant $10^{4} \mathrm{~Hz}$ frequency, showing the $\gamma$ transition and the $\gamma$ and $\gamma^{\prime}$ transitions for the pristine and bromine doped PAN, respectively

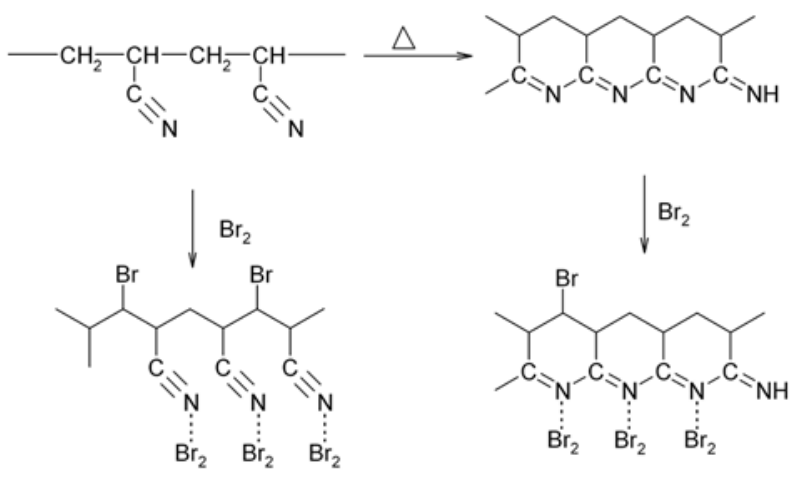

Figure 3. Chemical reactions that pertain to the material systems of Figure 1, showing polymerization of a sequence of pending nitrile groups in a PAN chain segment to form a conjugated ladder structure and doping with bromine to produce electron transfer adducts

bromination time was established by XPS (not shown here) [6]). Finally, the combined effect of heat treatment at $250^{\circ} \mathrm{C}$ and bromine doping is presented in Figure 1d; in this case the $\gamma$ process disappears altogether, indicating that even the $-\mathrm{Br}$ side groups, if formed by bromination, are locked in place by the ladder structure.

The chemical reactions that pertain to the results in Figure 1 and to the corresponding arguments can be presented schematically as follows (Figure 3). A sequence of pending nitrile groups in a PAN chain segment can polymerize to form a conjugated ladder structure of restricted mobility. Doping with bromine produces bulkier electron transfer adducts with either the nitrile/imine groups. Bromination of the main backbone can produce $\mathrm{C}-\mathrm{Br}$ covalent bonds in the two systems. (It is apparent that the weight increase by doping is accounted for by either or both $\mathrm{Br}$ forms.)

An additional expression of the effects of bromine doping and nitrile polymerization is the activation energy of the $\gamma$ process. It is anticipated that both effects produce increased activation energy as both generate excessive restrictions on chain segment movement. Figures $4 \mathrm{a}$ and $5 \mathrm{a}$ present plots of the dielectric loss as a function of frequency for a number of selected example temperatures for PAN and heat-treated PAN, respectively. In both, the peak of the $\gamma$ process is shifted to higher frequencies as the temperature is increased. This shift sets the basis for an Arrhenius type functionality, wherein the logarithm of the relaxation time is a linear function of the reciprocal peak temperature. Accordingly, Figures $4 b$ and $5 b$ present the Arrhenius plots of the 

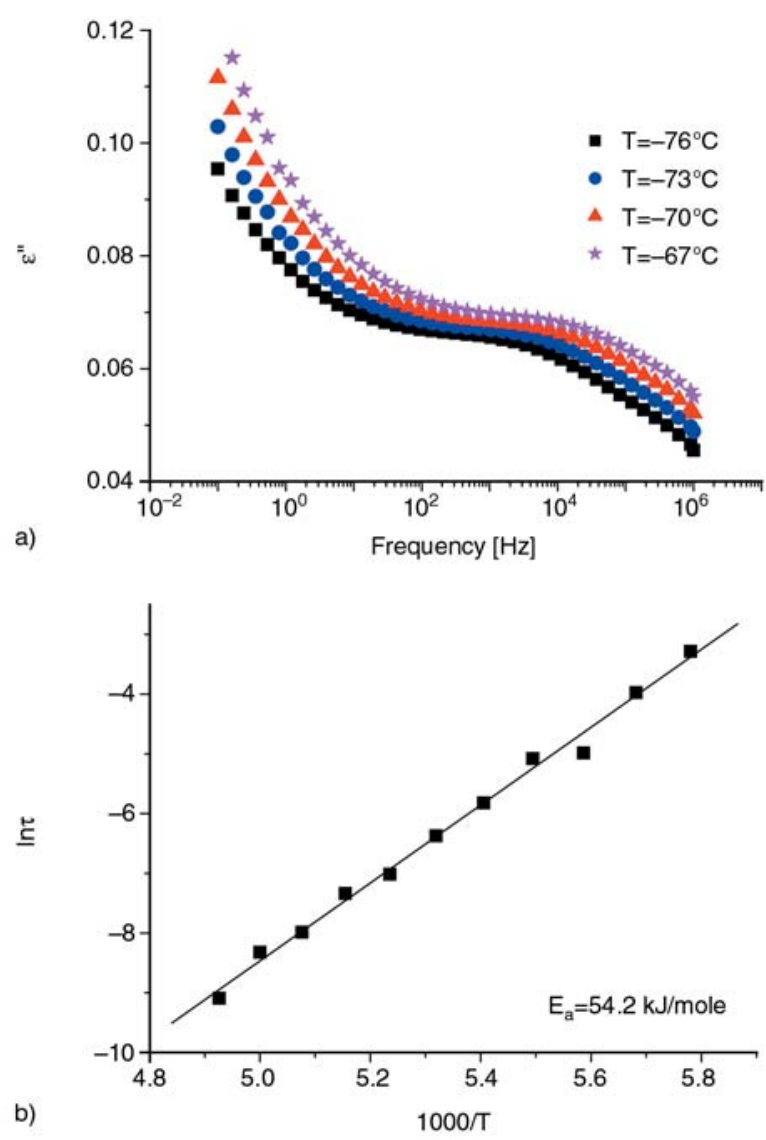

Figure 4. Plots for PAN of: (a) the dielectric loss as a function of frequency in a number of selected temperatures; (b) the logarithm of relaxation time as a function of the reciprocal peak temperature

same samples. The data comply with the model, exhibiting linear behavior of high statistically significant correlation coefficients and yielding (from the slopes of the lines) activation energies of 54.2 and $62.3 \mathrm{~kJ} / \mathrm{mole}$ in the temperature range from -100 to $-30^{\circ} \mathrm{C}$, for PAN and heat-treated PAN, respectively. Obviously, the activation energy of the $\gamma$ process in the partially polymerized nitrile system is $15 \%$ higher, indicative of the restricted movement.

Plots of the dielectric loss as a function of frequency of the bromine-doped PAN for a few selected temperatures, chosen from the temperature range where both $\gamma$ and $\gamma^{\prime}$ appear, are shown in Figure 6. The Arrhenius based activation energies of these processes were calculated (over the full temperature range) to be 64.4 and $59.5 \mathrm{~kJ} / \mathrm{mole}$, respectively. It is seen that the formation of brominenitrile adducts, expressed by splitting of the original $\gamma$ process, results in higher activation energies
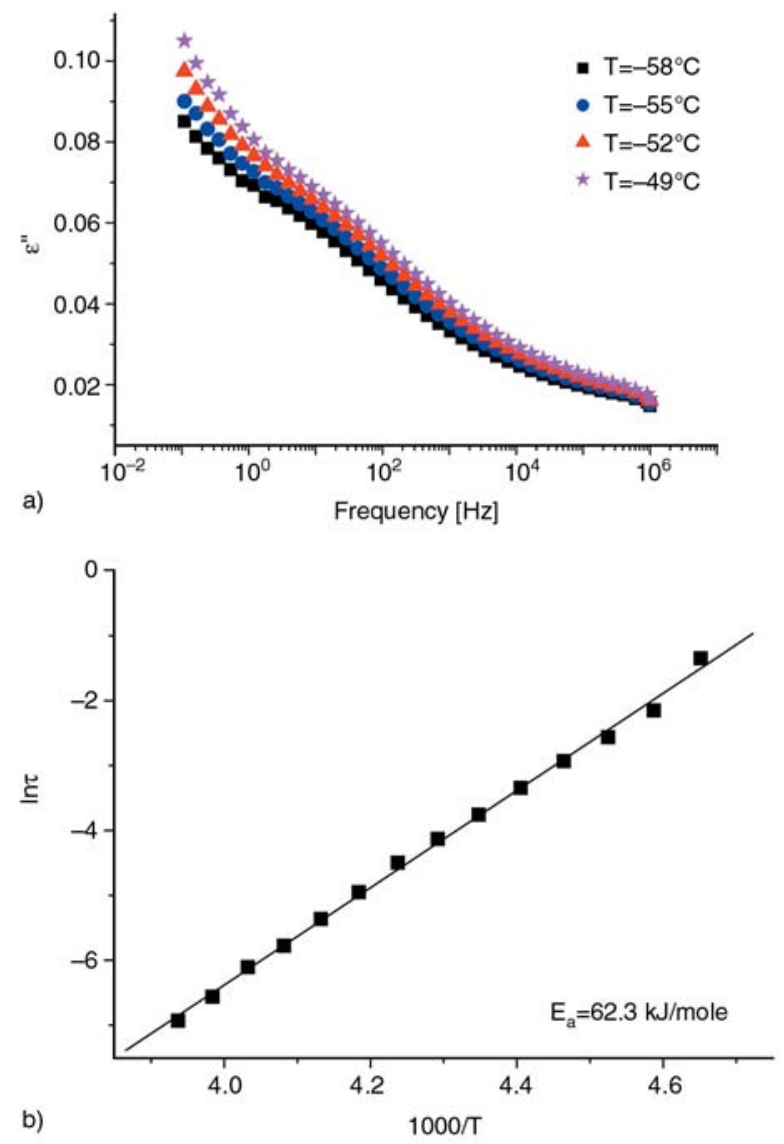

Figure 5. Plots for heat-treated PAN of: (a) the dielectric loss as a function of frequency in a number of selected temperatures; (b) the logarithm of relaxation time as a function of the reciprocal peak temperature

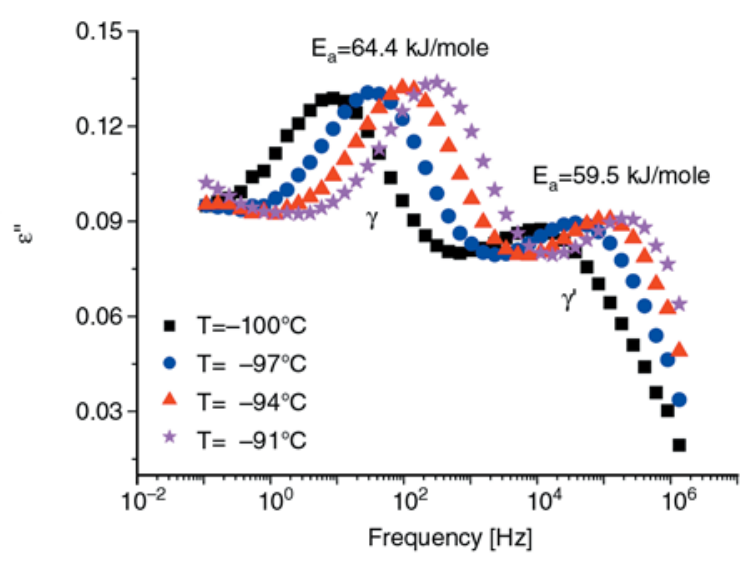

Figure 6. Plots of the dielectric loss $\varepsilon$ " as a function of frequency in a number of selected temperatures from the temperature range where both $\gamma$ and $\gamma^{\prime}$ appear, indicating the activation energies of the two processes

in the two processes and not only in the $\gamma^{\prime}$ process that is assigned to the adduct. 


\section{Conclusions}

The $\gamma$ process in PAN appears in the lower temperature range and the tail of this relaxation extends down to $-100^{\circ} \mathrm{C}$. It denotes a relaxation that is associated with movement of pending nitrile groups at activation energy of $54.2 \mathrm{~kJ} / \mathrm{mole}$. The restriction of movement associated with the thermal treatment that produces the conjugated imine system as part of a ladder structure results in diminishing of the $\gamma$ process and in raising its activation energy by $15 \%$. Doping with bromine produces two consequences that relate to the $\gamma$ process, namely, the process is spilt into two and the activation energies increase by $10-15 \%$. The appearance of the $\gamma^{\prime}$ process is taken as significant evidence for the formation of nitrile-bromine charge transfer complexes.

\section{References}

[1] Cohen D., Marom G., Zilkha A.: Physical properties of polyacrylonitrile fibres treated with organotin compounds. European Polymer Journal, 12, 795-800 (1976).

[2] Brokman A., Weger M., Marom G.: Electrical conductivity of halogen doped stabilized polyacrylonitrile. Polymer, 21, 1114-1115 (1980).
[3] Wang Y., Yang Q., Shan G., Wang C., Du J., Wang S., Li Y., Chen X., Jing X., Wei Y.: Preparation of silver nanoparticles dispersed in polyacrylonitrile nanofiber film spun by electrospinning. Materials Letters, 59, 3046-3049 (2005).

[4] Vaisman L., Larin B., Davidi I., Wachtel E., Marom G., Wagner H. D.: Processing and characterization of extruded drawn MWNT-PAN composite filaments. Composites, Part A, 38, 1354-1362 (2007).

[5] Vaisman L.: The reinforcement effect in carbon nanotube based polymer composites. Ph.D. Thesis, The Hebrew University of Jerusalem (2007).

[6] Cohen N.: Molecular processes and electronic properties of PAN doped with bromine. M.Sc. Thesis, The Hebrew University of Jerusalem (2007).

[7] Ward I. M., Hadely D. W.: An introduction to the mechanical properties of solid polymers. John Wiley and Sons, Chichister (1993).

[8] Sawai D., Kanamoto T., Yamazaki H., Hisatani K.: Dynamic mechanical relaxations in PAN with different stereoregularities. Macromolecules, 37, 2839 2846 (2004).

[9] Feldman Y., Kozlovich N., Nir I., Garti N.: Dielectric relaxation in sodium bis(2-ethylhexyl)sulfosuccinatewater-decane microemulsions near the percolation temperature threshold. Physical Review E, 51, 478 491 (1995).

[10] Li J., Vaisman L., Marom G., Kim J. K.: Br treated graphite nanoplatelets for improved electrical conductivity of polymer composites. Carbon, 45, 744-750 (2007). 